\title{
A modified protocol for the isolation, culture, and cryopreservation of rat embryonic neural stem cells
}

\author{
HENGXING ZHOU ${ }^{1,2^{*}}$, HUAN YANG $^{3 *}$, LU LU $^{1,2^{*}}$, XUEYING LI ${ }^{4 *}$, BIN PAN $^{1,2}$, \\ ZHENG FU ${ }^{4}$, TIANCI CHU ${ }^{5}$, JUN LIU ${ }^{1,2}$, YI KANG ${ }^{1,2}$, LU LIU $^{1,2}$, GUANGZHI NING $^{1,2}$, \\ WENYUAN DING ${ }^{6}$, PING WU ${ }^{7}$, XIAOHONG KONG ${ }^{8}$ and SHIQING FENG ${ }^{1,2}$
}

${ }^{1}$ Department of Orthopedics, Tianjin Medical University General Hospital, Tianjin 300052;

${ }^{2}$ Key Laboratory of Post-Neuroinjury Neuro-Repair and Regeneration in Central Nervous System,

Tianjin Neurological Institute, Ministry of Education and Tianjin City, Tianjin 300052;

${ }^{3}$ Department of Orthopedics, The Affiliated Hospital of Xuzhou Medical University, Xuzhou, Jiangsu 221006;

${ }^{4}$ Key Laboratory of Immuno Microenvironment and Disease of The Educational Ministry of China, Department of Immunology, Tianjin Medical University, Tianjin 300070, P.R. China; ${ }^{5}$ Department of Pediatrics, Kosair Children's Hospital Research Institute, University of Louisville School of Medicine, Louisville, KY 40202, USA; ${ }^{6}$ Department of Orthopedics, The Third Hospital of Hebei Medical University, Shijiazhuang, Hebei 050051, P.R. China;

${ }^{7}$ Department of Neuroscience and Cell Biology, University of Texas Medical Branch, Galveston, TX 77555-1043, USA;

${ }^{8}$ School of Medicine, Nankai University, Tianjin 300071, P.R. China

Received October 31, 2018; Accepted May 16, 2019

DOI: $10.3892 / \mathrm{etm} .2020 .9285$

\begin{abstract}
Neural stem cells (NSCs) are characterized by their potential for self-renewal and ability to differentiate into neurons, astrocytes, and oligodendrocytes. They are of great value to scientific studies and clinical applications. Culturing NSCs in vitro is important for characterizing their properties under controlled environmental conditions that may be modified and monitored accurately. The present study explored a modified, detailed and efficient protocol for the isolation, culture and cryopreservation of rat embryonic NSCs. In particular, the viability, nestin expression, and self-renewal and multi-differentiation capabilities of NSCs cryopreserved for various periods of time (7 days, or 1, 6 or 12 months) were characterized and compared. Rat embryonic NSCs were successfully obtained and maintained their self-renewal
\end{abstract}

Correspondence to: Professor Xiaohong Kong, School of Medicine, Nankai University, 94 Weijin Road, Nankai, Tianjin 300071, P.R. China

E-mail: xiaohong_kong@163.com

Professor Shiqing Feng, Department of Orthopedics, Tianjin Medical University General Hospital, 154 Anshan Road, Heping, Tianjin 300052, P.R. China

E-mail: sqfeng@tmu.edu.cn

*Contributed equally

Key words: neural stem cells, primary culture, multipotency, self-renewal, cryopreservation and multipotent differentiation capabilities even following long-term cryopreservation (for up to 12 months).

\section{Introduction}

Neural stem cells (NSCs) are multipotent cells capable of self-renewal and differentiation into neurons, astrocytes and oligodendrocytes (1-8). They have exhibited potential for the study of neural development, disease modeling, and drug and toxin screening, and they have therapeutic potential for neural disorders, including spinal cord injury and a number of other neurodegenerative diseases (2-5,7-9). As a complement to in vivo studies, the successful in vitro primary culture of NSCs is important, as it provides a powerful tool for determining the properties of NSCs under controlled environmental conditions that may be modified and monitored accurately (10). However, the complicated procedures required for NSC isolation and culture, and inconsistent operational details, not only restrict the yield of viable NSCs but also impede the intraand inter-laboratory comparison and reproducibility of experimental results $(3,11,12)$. Therefore, it is essential to provide a detailed and refined protocol that may be readily reproduced for the study of embryonic NSCs. The present study described a modified, detailed and feasible protocol for the isolation, culture and cryopreservation of rat embryonic NSCs. Compared with other previous protocols, the colorless high-glucose medium and a sequential digestion strategy were the primary modifications. In addition, the viability, nestin expression, and capability for self-renewal and multi-differentiation of NSCs cryopreserved for different time periods ( 7 days, or 1,6 or 12 months) were determined. 


\section{Materials and methods}

Animals. All animal procedures were approved by the Ethics Committee of Tianjin Medical University and complied with the United States of America National Institutes of Health Guide for the Care and Use of Experimental Animals and the Society for Neuroscience Use of Animals in Neuroscience Research guidelines $(13,14)$. A specific-pathogen-free Sprague Dawley female rat (age, 3-5 weeks; weight, 250 $\pm 30 \mathrm{~g}$; $\mathrm{n}=1$ ), pregnant at the embryonic age of 15.5 days (E15.5), was obtained from the Radiation Study Institute Animal Center at Tianjin Medical University (Tianjin, China), and was housed in a controlled environment $\left(23 \pm 1^{\circ} \mathrm{C}\right)$ under a 12-h light/dark cycle with free access to food and water. NSCs may be isolated as early as E10.5 in rats. In the present study, E15.5 rats were selected as the sources for NSC culture as NSCs were most abundant at E15.5, which is approximately the onset of neurogenesis (15).

Uterus anatomy and isolation. The major steps of the protocol modified in the present study are summarized in the flow diagram presented in Fig. 1. A set of microdissection instruments were autoclaved prior to use for uterus removal, with two sets of microdissection instruments for brain hemisphere dissection. The pregnant rats were sacrificed by cervical dislocation, immersed promptly into $-20^{\circ} \mathrm{C} 70 \%$ ethanol for $5 \mathrm{~min}$ for disinfection, and positioned in a sterilized dissecting tray with the abdomen facing upward. The abdominal fur of each rat was shaved rapidly, and the skin was re-sterilized using povidone-iodine swabs (Shandong Lierkang Medical Technology Co., Ltd.) to increase the aseptic conditions; any loose fur was removed from the skin. A sterile drape was used to cover the rat (Fig. 2A), and the abdominal skin above the genitalia was grasped using large straight forceps (Shanghai Medical Instruments Co., Ltd.). Then, an incision from the lower abdomen upward to the chest was made with large straight surgical scissors (Shanghai Medical Instruments Co., Ltd.). The peritoneal cavity was exposed sufficiently with hemostatic forceps (Shanghai Medical Instruments Co., Ltd.) to view the uterus. The intact uterus was held with a different pair of large straight forceps at the junction with the vagina (Fig. 2B), pulled up slightly, and isolated using a pair of small scissors (Shanghai Medical Instruments Co., Ltd.). The mesometrium and blood vessels were removed from the uterus with care to avoid bacterial contamination caused by damaging the intestines. Then, the uterus was transferred quickly to a $100-\mathrm{mm}$ dish (BD Biosciences) and rinsed 3 times with a $4^{\circ} \mathrm{C}$ PBS solution (Sigma-Aldrich; Merck KGaA) supplemented with $1 \%$ penicillin/streptomycin $(\mathrm{P} / \mathrm{S}$; Invitrogen; Thermo Fisher Scientific, Inc.; cat no., 15140148; Fig. 2C and D). The carcass was immediately disposed following uterus removal. While grasping one horn of the uterus in one hand using microforceps (Shanghai Medical Instruments Co., Ltd.), the uterus was opened by careful incision of the top of the uterus using microscissors (Shanghai Medical Instruments Co., Ltd.) placed in the other hand, under a SZX16 light stereomicroscope (Olympus Corporation; Fig. 2E), and then the amniotic membrane was exposed (Fig. 2F). Then, the amniotic membrane was removed and the placenta and embryo was exposed (Fig. 2G). Then the placenta was cut and the embryos (Fig. $2 \mathrm{H}$ ) were excised and rinsed 3 times with high-glucose Dulbecco's Modified Eagle's
Medium without phenol red (DMEM-HG; Gibco; Thermo Fisher Scientific, Inc.; cat no., 31053028) with $1 \%$ P/S. At this point, the embryos were assessed, and the color and number of live births were recorded. Embryos that appear malformed, too small or too large with respect to the expected gestational age were discarded. Then, live embryos (5-7 embryos each; Fig. 2I) were transferred into several $50 \mathrm{ml}$ centrifuge tubes (BD Biosciences) containing $4^{\circ} \mathrm{C}$ DMEM-HG with $1 \% \mathrm{P} / \mathrm{S}$. These embryos were subjected to additional brain dissection on ice, 1 centrifuge tube at a time, while the remaining embryos were kept in the refrigerator.

Ethanol $\left(-20^{\circ} \mathrm{C}, 70 \%\right)$ was used to not only sterilize the pregnant rats but also rapidly cool embryos and decrease the robust consumption of oxygen and energy by tissues $(7,10)$. (2) Fur is a source of contamination, and it may adhere to the peritoneal cavity, the scissors or brain tissues (3); therefore, some measures were taken to avoid this. For example, the abdominal fur was thoroughly shaved, the abdominal skin was carefully re-sterilized to remove any loose fur, and the rat was covered by a sterile drape. These precautions decreased the chance of fur contaminating the primary culture. However, if some fur adhered to the scissors accidently, the surgical instruments were rinsed using $70 \%$ ethanol and either allowed to dry or rinsed with PBS before resuming the experiment, as ethanol would fix the tissues $(10,16)$. (3) DMEM-HG without phenol red was selected as it provides a clear operative view for tissue dissection (17).

Brain dissection. Straight microforceps (held in the non-dominant hand) were used to grasp the body of each embryo, while a pair of microscissors (held in the dominant hand) was used to cut the head of each embryo at the level of the cervical spinal cord (Fig. 2J). All heads were collected and transferred promptly into a $100-\mathrm{mm}$ dish containing $4^{\circ} \mathrm{C}$ DMEM-HG with $1 \% \mathrm{P} / \mathrm{S}$ on ice. Then, they were transferred and dissected individually in a second dish containing $4^{\circ} \mathrm{C}$ DMEM-HG with $1 \% \mathrm{P} / \mathrm{S}$ on ice under a stereomicroscope (Fig. $2 \mathrm{~K}$ ). A pair of straight microforceps (held in the non-dominant hand) was used to steadily grasp the head of each embryo by placing the tip of the microforceps vertically along the caudal side of each ear, while the other pair of forceps (held in the dominant hand) was inserted into the nose (microforceps tip up) to peel off the skin with an expansion force through simultaneous bilateral tissue removal (Fig. 2L). The skull and dura mater were dissected by inserting the straight microforceps at the intersection of the lambda suture and then removing layer by layer to expose the cerebral hemispheres. Then, the entire cerebral hemispheres were excised and transferred gently using curved microforceps, tip up, (Shanghai Medical Instruments Co., Ltd.) to a $100-\mathrm{mm}$ dish containing $4^{\circ} \mathrm{C}$ DMEM-HG with $1 \% \mathrm{P} / \mathrm{S}$ on ice. All heads were dissected until all cerebral hemispheres were harvested.

Removal of the pia mater and blood vessels. An additional set of microdissection instruments was used to remove the pia mater and blood vessels of the cerebral hemispheres. These samples were dissected and individually transferred to another dish containing $4^{\circ} \mathrm{C}$ DMEM-HG with $1 \% \mathrm{P} / \mathrm{S}$ on ice under the stereomicroscope (Fig. 2M). A pair of straight microforceps (held in the non-dominant hand) was used to steadily grasp the 


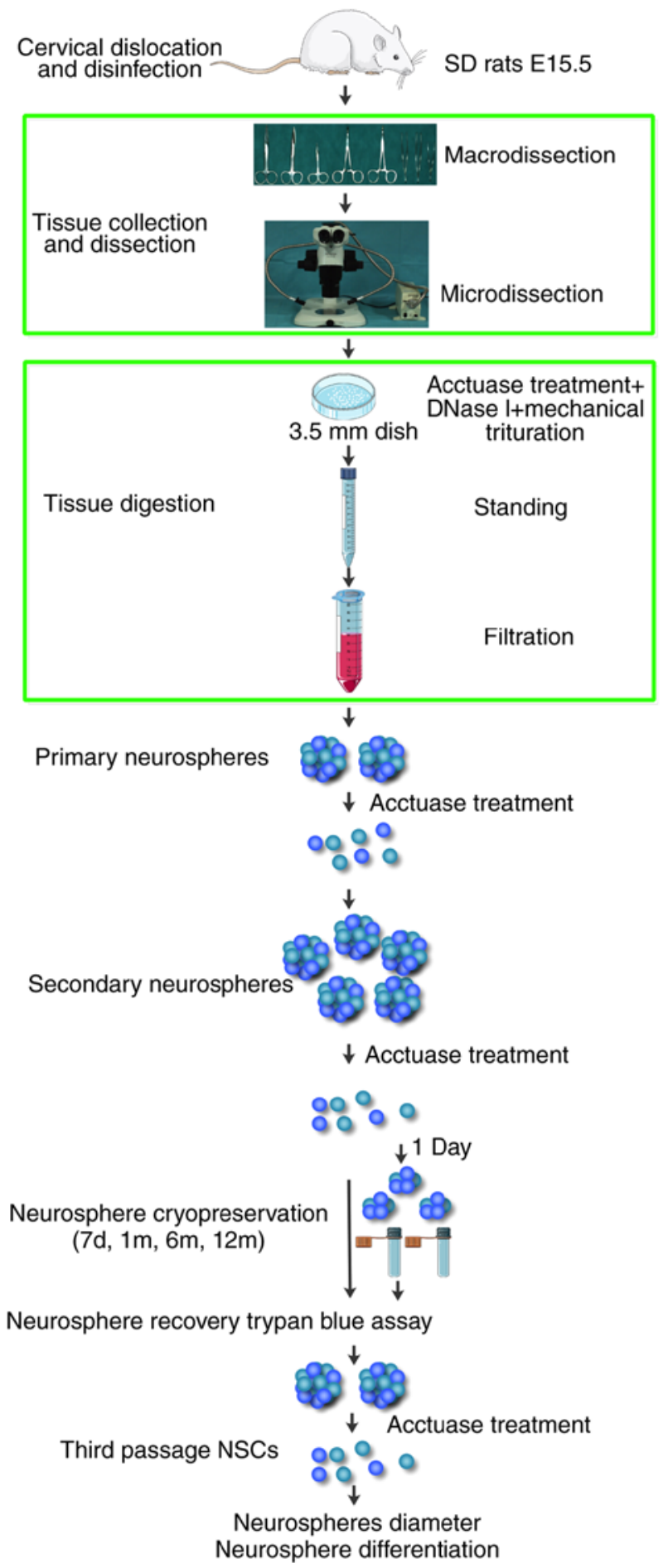

Figure 1. A schematic of the protocol steps.

cerebral hemisphere, while the other pair (held in the dominant hand) was used to carefully peel off the pia mater and blood vessels (Fig. 2N-P). The dissected cerebral hemisphere tissues were transferred to a separate dish containing $4^{\circ} \mathrm{C}$ DMEM-HG with $1 \% \mathrm{P} / \mathrm{S}$ on ice until all cerebral hemispheres were dissected. To facilitate the operation, it was necessary to periodically replace the dish filled with the stripped pia mater and blood vessels.

Sequential digestion of the dissected tissues from each hemisphere and preparation of cell suspensions. The dissected cerebral hemispheres were minced on ice using a pair of microscissors into small, even pieces $\left(\sim 1 \mathrm{~mm}^{3}\right)$ under the stereomicroscope for $\sim 1 \mathrm{~min}$. The minced tissues were then carefully transferred into a $15-\mathrm{ml}$ centrifuge tube (BD Biosciences) using a pre-wetted, fire-polished glass Pasteur pipette (Yangcheng Shuangfeng Glassware Co., Ltd), and then centrifuged for $5 \mathrm{~min}$ at $200 \mathrm{x} \mathrm{g}$ (Heraeus Multifuge X1; Thermo Scientific, Inc.) at $37^{\circ} \mathrm{C}$. The supernatant was then carefully removed, and 3-5 $\mathrm{ml}$ pre-warmed accutase solution (Gibco: Thermo Fisher Scientific, Inc.; cat no., 00-4555-56) with 20 units/ml deoxyribonuclease I (DNase I; cat. no., LS002060; Worthington Biochemical Corporation), was added to the precipitated tissues. This was adjusted according to the number of pups used. Following gentle trituration, the tissue was transferred to a $35 \mathrm{~mm}$ sterile dish (BD Biosciences) using a pre-wetted, fire-polished glass Pasteur pipette and incubated for $20 \mathrm{~min}$ at $37^{\circ} \mathrm{C}$. During the incubation, the tissue was gently triturated with a glass Pasteur pipette 7-8 times every 5 min. All suspended and undissociated tissues were collected into a $15 \mathrm{ml}$ centrifuge tube and held for $2 \mathrm{~min}$; then, three-fourths of the supernatant was carefully aspirated and passed through a $70 \mu \mathrm{m}$ cell strainer (BD Biosciences) into a 50-ml centrifuge tube containing $30 \mathrm{ml}$ DMEM-HG with $1 \% \mathrm{P} / \mathrm{S}$ to remove any cell clumps and tissue pieces. The tube was kept at room temperature (RT) until completion of the sequential digestion. Following the initial digestion, the precipitated tissue in the $15 \mathrm{ml}$ centrifuge tube was resuspended in $3 \mathrm{ml}$ accutase solution with 20 units $/ \mathrm{ml}$ DNase I for the second digestion. As with the first digestion, the enzymatic mix was pipetted 7-8 times every 5 min with a glass Pasteur pipette, and then aspirated and filtered through a $70-\mu \mathrm{m}$ cell strainer placed on the $50-\mathrm{ml}$ centrifuge tube containing the single-cell suspension from the first digestion. Following centrifugation for $5 \mathrm{~min}$ at $200 \mathrm{x} \mathrm{g}$ at $37^{\circ} \mathrm{C}$, the supernatant was discarded, and the cell pellet was resuspended in $20 \mathrm{ml}$ fresh serum-free medium [SFM; DMEM/Ham's F-12 (DMEM/F-12); Invitrogen: Thermo Fisher Scientific, Inc.; cat no., 11330032) containing $20 \mathrm{ng} / \mathrm{ml}$ basic fibroblast growth factor (bFGF; cat. no., 450-33; PeproTech, Inc.), $20 \mathrm{ng} / \mathrm{ml}$ epidermal growth factor (EGF; cat. no., 400-25; PeproTech, Inc.), $2.5 \mu \mathrm{g} / \mathrm{ml}$ heparin (cat. no., 2812; Tocris Bioscience), 2\% B-27 supplement (cat. no. 17504044), $1 \mathrm{mM}$ L-glutamine (cat. no., 25030149) and 1\% P/S (all from Invitrogen; Thermo Fisher Scientific, Inc.). Suspension aliquots $(10 \mu \mathrm{l})$ were mixed with $10 \mu 1$ Trypan blue (Sigma-Aldrich; Merck KGaA) at room temperature for $3 \mathrm{~min}$ to calculate cell viability using a hemocytometer and phase-bright IX71 microscope with a x10 objective (Olympus Corporation). The dissociated cells were seeded in uncoated $15 \mathrm{ml}$ T75 culture flasks (Corning Life Sciences) at a density of $2 \times 10^{5}$ cells $/ \mathrm{ml}$ and incubated at $37^{\circ} \mathrm{C}$ with $5 \% \mathrm{CO}_{2}$.

Compared with a glass Pasteur pipette, 1,000 $\mu$ l plastic pipette tips are considered superior for the discharge of cells, leading to lower cell viability; in addition, cells adhere to plastic tips more easily, leading to increased cell loss $(2,7,18,19)$. Therefore, a glass Pasteur pipette was used, and it was pre-wetted with a small amount of medium to prevent tissue from sticking to the glass surface. The total digestion time should not be $>40 \mathrm{~min}$; excessive and prolonged digestion may lead to fewer viable cells $(7,16,20)$. Fresh SFM could be kept 

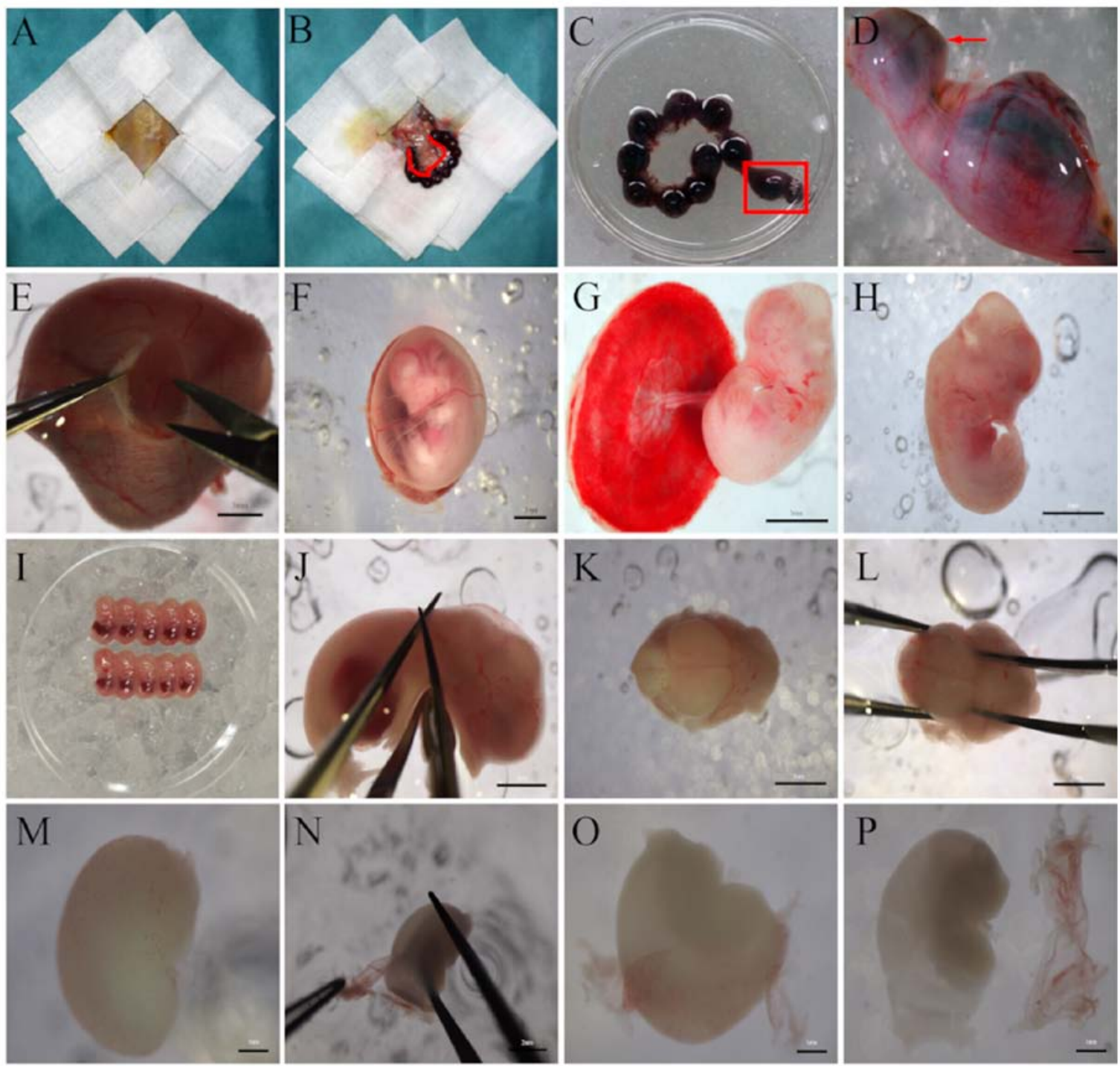

Figure 2. Macrodissection and microdissection processes used for brain collection. (A) The sacrificed pregnant rat was positioned on its back to expose the abdomen; the abdominal skin was then shaved and resterilized with povidone-iodine swabs. (B) The mesometrium and blood vessels were removed from the uterus (red line). (C) The uterus was then removed and rinsed. (D) High magnification image of C (arrow indicates malformed uterus). (E) While grasping one horn of the uterus in one hand using microforceps, the top of the uterus was carefully cut through with microscissors held in the other hand to open the uterus using a SZX16 light stereomicroscope. (F) The amniotic membrane was exposed. (G) The amniotic membrane was removed, and the placenta and embryo was exposed. (H) The embryo following removal of placenta and attached membranes. (I) The remaining embryos. (J) Straight microforceps (held in the non-dominant hand) were used to grasp the body of each embryo, while a pair of microscissors (held in the dominant hand) was used to cut the head of each embryo at the level of the cervical spinal cord. (K) The head was separated. (L) One pair of straight microforceps (held in the non-dominant hand) was used to steadily grasp each embryo head by placing the tip of the microforceps vertically along the caudal side of each ear, while the other pair of forceps (held in the dominant hand) was inserted into the nose (microforceps tip up) to peel off the skin with an expansion force through simultaneous bilateral tissue removal. (M) The cerebral hemispheres were harvested. (N) A pair of straight microforceps (held in the non-dominant hand) was used to steadily grasp the cerebral hemisphere, while the other pair (held in the dominant hand) was used to carefully peel off the pia mater and blood vessels. (O) A hemisphere with part of the pia mater and blood vessels. (P) A hemisphere was completely peeled off the pia mater and blood vessels.

at $4^{\circ} \mathrm{C}$ for a maximum of 1 week to avoid significant decreases in the biological effect of critical growth factors, including bFGF, EGF and B-27, which were added to the medium over time $(16,20-22)$. Therefore, bFGF, EGF and B-27 were added to the medium on the day of the experiments to obtain optimal results. In addition, the SFM was pre-warmed prior to use, to avoid the cell damage and low cell viability generated by sudden temperature change (16). (4) To prevent neurospheres from adhering to the bottom of the culture flasks, ultra-low attachment flasks (Corning Inc.) were used, and tapped them several times every week, with the exception of the first 3 days after initial plating $(19,23)$.
Medium replacement and cell passaging. After 3 days of culture, medium containing neurospheres was transferred to a $50 \mathrm{ml}$ centrifuge tube using a glass Pasteur pipette. Following centrifugation for $5 \mathrm{~min}$ at $200 \mathrm{x}$ g at $37^{\circ} \mathrm{C}$, the supernatant was transferred into a new $50 \mathrm{ml}$ centrifuge tube. A total of one-half of the supernatant was replaced with fresh SFM, and the resulting mixture was used to resuspend the pelleted cells, which were then seeded into new culture flasks and incubated at $37^{\circ} \mathrm{C}$ with $5 \% \mathrm{CO}_{2}$.

Passaging was performed after 7-8 days in culture. Neurospheres were handled similarly as aforementioned, with the exception of the resuspension of cell pellets in pre-warmed 
accutase solution. The enzyme-cell suspension was incubated at $37^{\circ} \mathrm{C}$ for $10 \mathrm{~min}$, and then triturated 5 times using a $2 \mathrm{ml}$ disposable needle (23 Gauge, 0.6x32 mm; BD Biosciences). These enzymatically and mechanically dissociated cells were then transferred to a $50 \mathrm{ml}$ centrifuge tube containing $30 \mathrm{ml}$ pre-warmed DMEM-HG. Following centrifugation for $5 \mathrm{~min}$ at $200 \mathrm{x} \mathrm{g}$ at $37^{\circ} \mathrm{C}$, the cell pellets were resuspended in fresh SFM. An aliquot of cell suspension $(10 \mu \mathrm{l})$ was mixed with $10 \mu 1$ Trypan blue at room temperature for 3 min to calculate cell viability using a hemocytometer under bright-phase microscopy with a 10 objective. Cells were plated into culture flasks at a density of $2 \times 10^{5}$ cells $/ \mathrm{ml}$ and incubated at $37^{\circ} \mathrm{C}$ with $5 \% \mathrm{CO}_{2}$. A total of one-half of the medium was replaced with fresh SFM 3 days after passaging, and the cells were sub-cultured after a total of 7-8 days in culture. The majority of the second-passage neurospheres were cryopreserved, as described subsequently, and the third-passage neurospheres (non-cryopreserved or cryopreserved) were used in the subsequent experiments.

During the first 3 days after initial plating, cells adjust to their new environment, start communicating with neighboring cells and begin to form neurospheres. Therefore, the culture flasks could not be moved during the first 3 days of culture $(17,21,22)$. If neurospheres became attached to the culture flasks during harvest, they were detached by either tapping on the sides of the flasks or aspirating them with medium. For cell dissociation, it was critical to aspirate all the enzymatic suspension into the syringe and discharge it slowly through the needle to ensure uniform and sufficient trituration (21).

Cryopreservation and recovery of neurospheres. The primary neurospheres were dissociated with pre-warmed accutase, resuspended in fresh SFM, and cultured at $37^{\circ} \mathrm{C}$ with $5 \%$ $\mathrm{CO}_{2}$ for $24 \mathrm{~h}$ prior to cryopreservation upon the formation of small cellular spheres. A freezing container (Nalgene ${ }^{\mathrm{TM}}$ Cryo; Thermo Fisher Scientific, Inc.) was placed at RT and filled with $100 \%$ isopropyl alcohol (Tianjin Wanding Chemicals Co., Ltd.); freezing medium was prepared [90\% SFM and $10 \%$ dimethyl sulfoxide (DMSO); cat. no., D2650; Sigma-Aldrich; Merck KGaA] protected from light sources. Culture medium containing small neurospheres was transferred into a $50 \mathrm{ml}$ centrifuge tube. Following centrifugation for $5 \mathrm{~min}$ at $200 \mathrm{xg}$ at $37^{\circ} \mathrm{C}$, the supernatant was decanted, and the cell pellet was gently resuspended in freshly prepared freezing medium at a concentration of 5-10x $10^{6}$ cells $/ \mathrm{ml}$ using a glass Pasteur pipette. The suspension was immediately aliquoted $(1 \mathrm{ml})$ into labeled cryovials, and the vials were immediately placed into the container. Following storage at $-80^{\circ} \mathrm{C}$ for $24 \mathrm{~h}$, the cell aliquots were transferred into liquid nitrogen for storage for 7 days, or 1,6 or 12 months.

For the recovery of cryopreserved NSCs, 1 cryovial per time point (7 days, or 1, 6 or 12 months) was removed from the liquid nitrogen as quickly as possible, promptly placed into a water bath at $37^{\circ} \mathrm{C}$, and swirled quickly to accelerate the thawing process. The entire cryovial was sprayed with $70 \%$ ethanol and moved to a clean bench; then, the cell suspension was immediately aspirated in a controlled manner and added dropwise to a $50 \mathrm{ml}$ centrifuge tube containing $40 \mathrm{ml}$ pre-warmed DMEM-HG. Following centrifugation for $5 \mathrm{~min}$ at $200 \mathrm{x} \mathrm{g}$ at $37^{\circ} \mathrm{C}$, the cell pellet was resuspended in $40 \mathrm{ml}$ pre-warmed DMEM-HG for a second rinse and centrifugation $\left(5 \mathrm{~min}\right.$ at $200 \mathrm{x} \mathrm{g}$ at $37^{\circ} \mathrm{C}$ ). The cell pellet was then resuspended in $20 \mathrm{ml}$ fresh, pre-warmed SFM. The cell suspension was transferred into culture flasks and incubated at $37^{\circ} \mathrm{C}$ with $5 \% \mathrm{CO}_{2}$. A total of one-half of the medium was replaced after 3 days of culture, and cells were sub-cultured after 7-8 days of culture for additional experiments.

During the thawing process, the cryovials should not be not completely submerged in water, and care must be taken when swirling the cryovial to prevent contamination. The DMEM-HG and SFM were all pre-warmed to protect the NSCs from damage and low cell viability caused by the sudden temperature change that occurs when vulnerable post-thaw cells are added to the medium.

Differentiation of dissociated neurospheres. Induced differentiation is commonly applied to examine the multipotential differentiation capability of non-cryopreserved and cryopreserved NSCs $(16,23)$. Firstly, sufficient poly-L-lysine (PLL; molecular weight 150,000-300,000, 0.01\%; cat. no., P4832; Sigma-Aldrich; Merck KGaA) was added to coat the surface of 12 -well culture plates for $24 \mathrm{~h}$ in a $37^{\circ} \mathrm{C}$ incubator. Following removal of the PLL, the plates were washed 3 times with PBS and air-dried in the hood prior to use. The non-cryopreserved and cryopreserved secondary neurospheres (stored for 7 days, or 1, 6 or 12 months) were collected, dissociated, and seeded on PLL-coated 12-well culture plates at a density of $5 \times 10^{4} / \mathrm{cm}^{2}$ in differentiation medium (DMEM/F-12 medium containing B-27 supplement and 1\% FBS; cat. no., 16000044; Invitrogen; Thermo Fisher Scientific, Inc.) at $37^{\circ} \mathrm{C}$ with $5 \% \mathrm{CO}_{2}$ for up to 7 days. The differentiation medium was changed every 3 days. To detect astrocyte, neuron and oligodendrocyte differentiation, the cells were immunostained with glial fibrillary acidic protein (GFAP), neuron-specific class III beta-tubulin ( $\beta$-III-tubulin) and cyclic nucleotide phosphodiesterase (CNPase) antibodies, respectively (see details in the Immunocytochemistry section).

PLL coating may be performed for $2-24 \mathrm{~h}$ at $37^{\circ} \mathrm{C}$, and PLL-coated plates may be stored at RT for at least 1 week $(7,10,15,23)$. In the present study, to guarantee the maximum consistency of experimental conditions, the plates were coated with PLL at $37^{\circ} \mathrm{C}$ for $24 \mathrm{~h}$ prior to commencing the experiment.

\section{Immunocytochemistry}

Nestin. Non-cryopreserved and cryopreserved secondary neurospheres were dissociated and seeded on PLL-coated 12-well culture plates in a monolayer $\left(1 \times 10^{5} \mathrm{cell} / \mathrm{s} / \mathrm{ml}\right)$ and maintained for 3 days in fresh SFM. Non-cryopreserved and cryopreserved secondary neurospheres were collected and plated on PLL-coated 12-well culture plates and cultured for $24 \mathrm{~h}$. Both the monolayer cells and neurospheres were fixed with 4\% paraformaldehyde (cat. no., P1110; Beijing Solarbio Science \& Technology Co., Ltd.) for $20 \mathrm{~min}$ at RT, washed with PBS and permeabilized with 0.3\% Triton X-100 (cat. no., T8787; Sigma-Aldrich; Merck KGaA) for 5 min. They were rinsed 3 times with PBS and blocked with 10\% normal goat serum (cat. no., PCN5000; Invitrogen; Thermo Fisher Scientific, Inc.) in PBS for $1 \mathrm{~h}$ at RT. Cells were then incubated 
with nestin primary antibody (cat. no., N5413; Sigma-Aldrich; Merck KGaA) at a 1:200 dilution in PBS overnight at $4^{\circ} \mathrm{C}$. After 3 washes, the cells were subsequently incubated with Alexa Fluor 555-conjugated goat anti-rabbit secondary antibody (cat. no., A-21429; Invitrogen: Thermo Fisher Scientific, Inc.) at a 1:500 dilution in PBS in the dark for $1 \mathrm{~h}$. Then, the cells were rinsed 3 times with PBS and incubated with DAPI $(1 \mathrm{mg} / \mathrm{ml}$ diluted in PBS; Cat No. D9542; Sigma-Aldrich; Merck KGaA) for $5 \mathrm{~min}$ at RT.

GFAP, $\beta$-III-Tubulin and CNPase. Non-cryopreserved and cryopreserved secondary neurospheres were passaged and seeded on PLL-coated 12-well culture plates in a monolayer and maintained for 7 days in fresh differentiation medium, as aforementioned. The protocols used for GFAP, $\beta$-III-tubulin, and CNPase immunostaining were similar to the aforementioned method for nestin immunolabeling, with the exception that differentiated cells were incubated with rabbit anti-GFAP (1:1,000; cat. no., ab7260; Abcam), anti- $\beta$-III-tubulin (1:500; cat. no., PRB-435P; Covance, Inc.), and anti-CNPase (1:100; cat. no., 5664; Cell Signaling Technology, Inc.).

All immunolabeled cells were examined under a fluorescence microscope (IX71). Images of 5 randomly selected fields were acquired at magnification, x20. Image-Pro plus 6.0 software (Media Cybernetics, Inc.) was used for the data analysis, and quantification of immunolabeled cells was presented as the percentage of specific marker-positive cells in comparison to the total number of DAPI-stained cells.

Trypan blue exclusion assay. Viable cells were counted using trypan blue exclusion assays in a hemocytometer $(16,17,21,23,24)$. Necrotic cells with compromised plasma membranes permit dye entry, whereas viable cells exclude the dye (24). Therefore, the proportion of necrotic cells is the percentage of trypan blue-positive cells, whereas cell viability is the ratio of the number of trypan blue-impermeable cells to the total number of cells (24). In brief, non-cryopreserved and cryopreserved secondary neurospheres were dissociated and resuspended, and then suspension aliquots (10 $\mu \mathrm{l})$ were mixed with $10 \mu \mathrm{l}$ of trypan blue solution (cat. no., T8154; Sigma-Aldrich; Merck KGaA) for cell counting using a hemocytometer (room temperature) under a light microscope with x20 magnification. The total and trypan blue-positive cell numbers were then determined by counting at least 200 cells per sample in a hemocytometer.

Determination of neurosphere diameter. Non-cryopreserved and cryopreserved secondary neurospheres were passaged and cultured in flasks with fresh SFM. NSCs (single and spheres) were observed by bright-phase microscopy, and images were captured from 5 randomly selected fields with a x 20 objective on the first, second, third and fourth days after passaging. The number and diameter of the spheres were determined using Image-Pro plus 6.0 software.

Statistical analysis. GraphPad Prism 5.0 (GraphPad Software, Inc.) was used for the statistical analysis. All data are presented as the mean \pm standard error of the mean of experimental replicates. Statistical differences between the non-cryopreserved (the control groups) and cryopreserved (the experimental groups) cells were determined using a one-way analysis of variance followed by Bonferroni post-hoc test. $\mathrm{P}<0.05$ were considered to indicate a statistically significant difference.

\section{Results}

Isolation and characterization of rat embryonic NSCs. A single-cell suspension was obtained directly following isolation, and these cells began to proliferate to form small, round cell clusters with clear boundaries, which loosely adhered to the bottom of the flask as observed under phase contrast microscopy. The diameter of the cell clusters ranged from 30-50 $\mu \mathrm{m}$ (Fig. 3A). On day 3 , the cell clusters grew larger, detached from the bottom of the flask and became suspended in the medium, forming neurospheres of 50-80 $\mu \mathrm{m}$ in diameter. These neurospheres were phase-bright and nearly perfectly spherical in shape, with sharp, phase-bright outer edges (Fig. 3B). On day 7, these neurospheres exhibited a translucent center and small cilia-like projections around the periphery with a diameter of $130-150 \mu \mathrm{m}$ (Fig. 3C), and were ready for dissociation and passaging. If allowed to proliferate further, the center of the spheres turned dark, and the spheres began to attach to the bottom of the flask on day 9 (Fig. 3D). Notably, numerous dead cells and debris were present in the primary culture, and they aggregated and formed pseudo-spheres, which were not spheroid in shape but were instead composed of small, phase-dark, irregularly shaped cells. However, following 2 passages, the majority of dead cells and debris were effectively eliminated.

To characterize these rat embryonic NSCs, the secondary neurospheres were first immunostained with nestin, a specific marker for NSCs. A total of two-thirds of the cells in the outer zone of the neurospheres were positive for nestin, whereas cells in the center were nestin-negative (Fig. 4A and B). To obtain a more accurate estimation of the proportion of nestin-positive NSCs in these 3D neurospheres, the neurospheres were dissociated into single cells, seeded on PLL-plated plates in SFM, and immunostained with nestin. As demonstrated in Fig. 4C, $94.40 \pm 0.63 \%$ of cells in the monolayer expressed nestin.

Next, the multipotent differentiation capability of NSCs was determined. According to the immunofluorescence staining, $66.45 \pm 4.52,25.74 \pm 2.31$ and $1.62 \pm 0.42 \%$ of the cells differentiated into GFAP-positive astrocytes, $\beta$-III-tubulin-positive neurons and CNPase-positive oligodendrocytes, respectively (Fig. 4D and F).

Effects of the cryopreservation duration on rat embryonic NSCs. To examine the effects of cryopreservation duration on rat embryonic NSCs, the cell viability, nestin expression, multipotentiality and diameter of non-cryopreserved NSCs and cryopreserved NSCs (7 days, or 1, 6 or 12 months) were further analyzed and compared.

Cell viability following cryopreservation. The trypan blue exclusion assay was performed to assess cell viability $(16,17,21,23,17,24)$. As indicated in Fig. 5, 73.99 \pm 1.14 , $74.23 \pm 1.29,73.85 \pm 1.26$ and $73.96 \pm 1.05 \%$ of the cells survived from cryopreservation for 7 days, or 1, 6 or 12 months, respectively. These data indicated that NSCs cryopreserved for 7 days, or 1, 6 or 12 months did not exhibit differences in cell viability following recovery (all $\mathrm{P}>0.05$ ). 

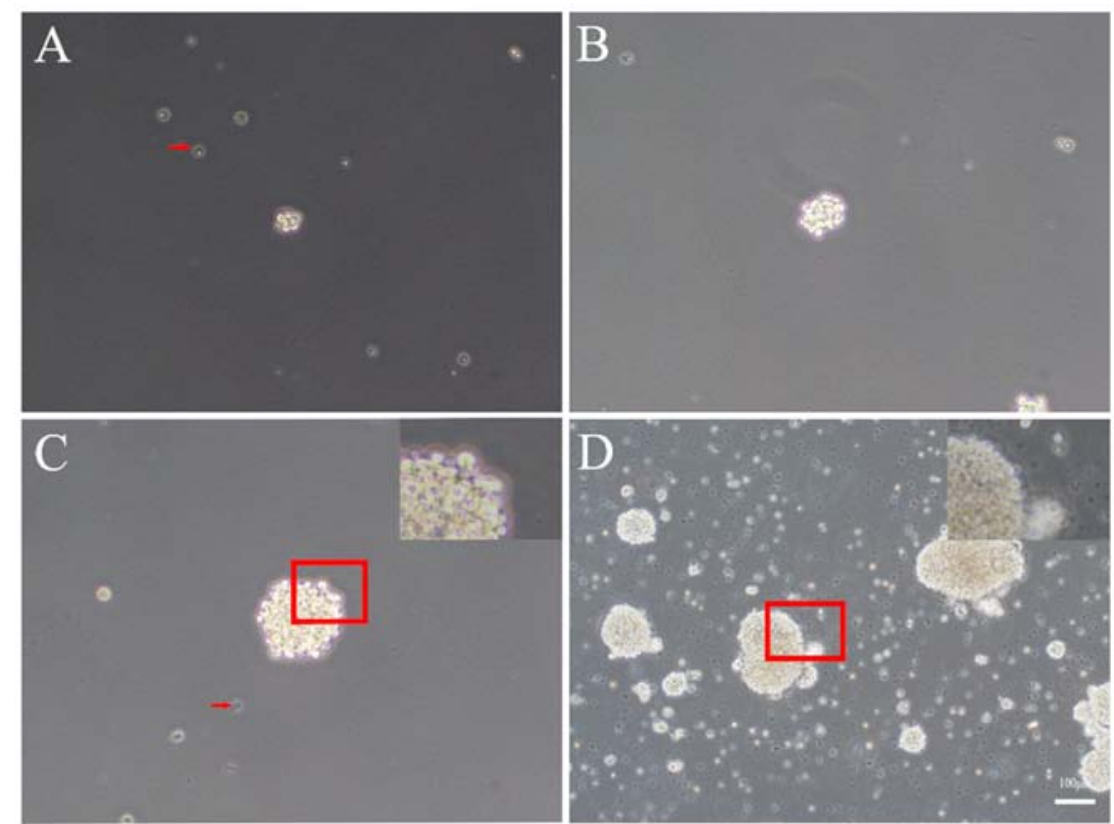

Figure 3. NSCs isolated from E15.5 rat cerebral hemispheres at different days during culture under bright-phase microscopy. (A) NSCs cultured for $24 \mathrm{~h}$ exhibited a clear boundary and loosely adhered to the bottom of the flask. (B) On day 3, neurospheres with a diameter of 50-80 $\mu \mathrm{m}$ had formed; they were phase-bright and nearly perfectly spherical in shape, with sharp, phase-bright outer edges. (C) On day 7, the neurospheres exhibited a translucent center and small cilia-like projections around the periphery; they now reached a diameter of 130-150 $\mu \mathrm{m}$. (D) Image of an unhealthy sphere on day 9 that displayed a dark area in the center; these neurospheres began to attach to the bottom of the flask. Scale bar=100 $\mu \mathrm{m}$. The inset on the top right corner is a magnification of the area labeled with a red box (magnification, $\mathrm{x} 2$ ).
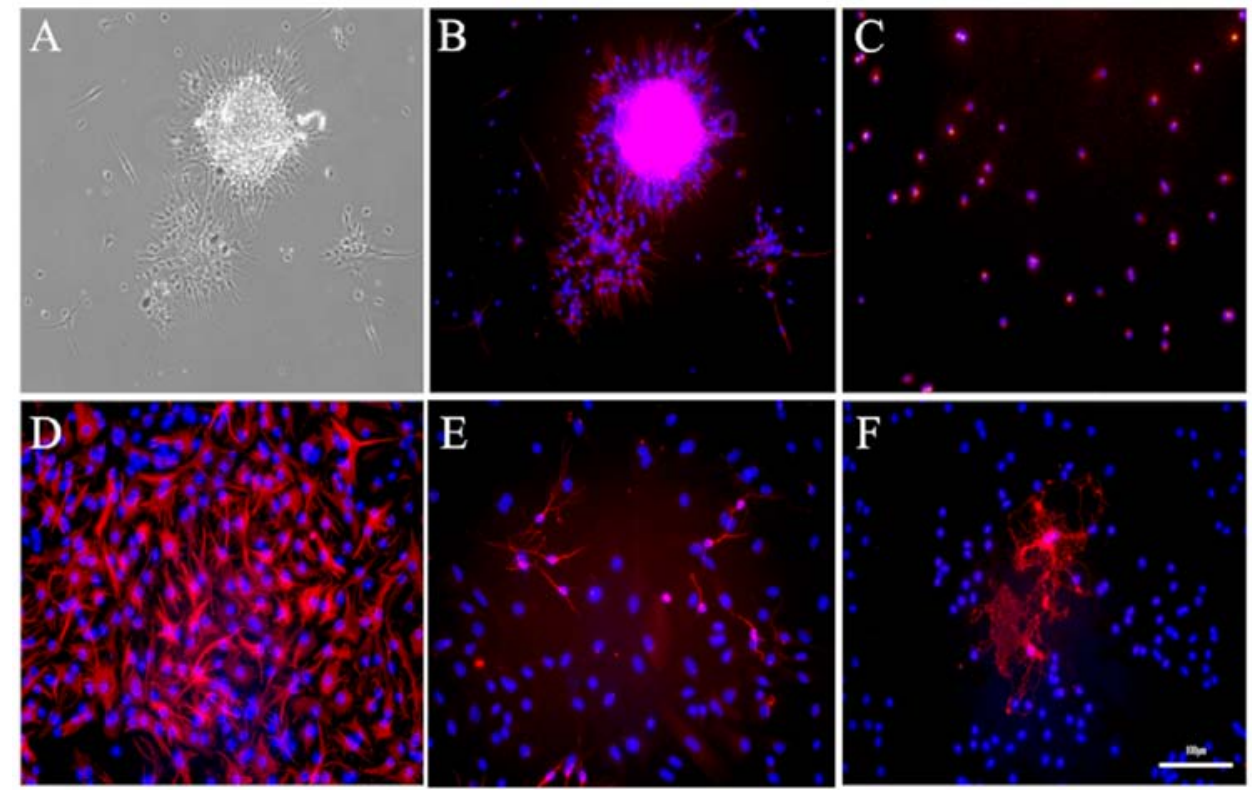

Figure 4. Identification of neurospheres and differentiation of dissociated neurospheres. (A) Phase contrast micrograph of 1-day culture of NSC neurospheres. (B) Representative image of neurospheres staining for Nestin. (C) Representative image of dissociated neurospheres staining for Nestin. (D) Representative image immunocytochemical staining for GFAP (red) and DAPI (blue) in NSC-derived astrocytes after 7 days of differentiation. (E) Representative image of immunocytochemical staining for $\beta$-III-tubulin (red) and DAPI (blue) in NSC-derived neurons after 7 days of differentiation. (F) Representative image of immunocytochemical staining for CNPase (red) and DAPI (blue) in NSC-derived oligodendrocytes after 7 days of differentiation. Scale bar=100 $\mu \mathrm{m}$.

ExpressionoftheNSCmarkernestinfollowingcryopreservation. To investigate the stem state of NSCs following different durations of cryopreservation, non-cryopreserved or cryopreserved secondary neurospheres were either directly immunostained for nestin or dissociated into single cells and then subjected to nestin immunostaining. Immunostaining of neurospheres indicated that all neurospheres expressed nestin (Fig. 6). Single-cell immunostaining additionally confirmed that $94.01 \pm 0.71,94.36 \pm 0.68,93.51 \pm 0.56$ and $94.27 \pm 0.63 \%$ of the cells expressed nestin in the recovered NSCs cryopreserved for 7 days, or 1, 6 or 12 months, respectively. No significant differences were observed in the percentage of nestin-positive 

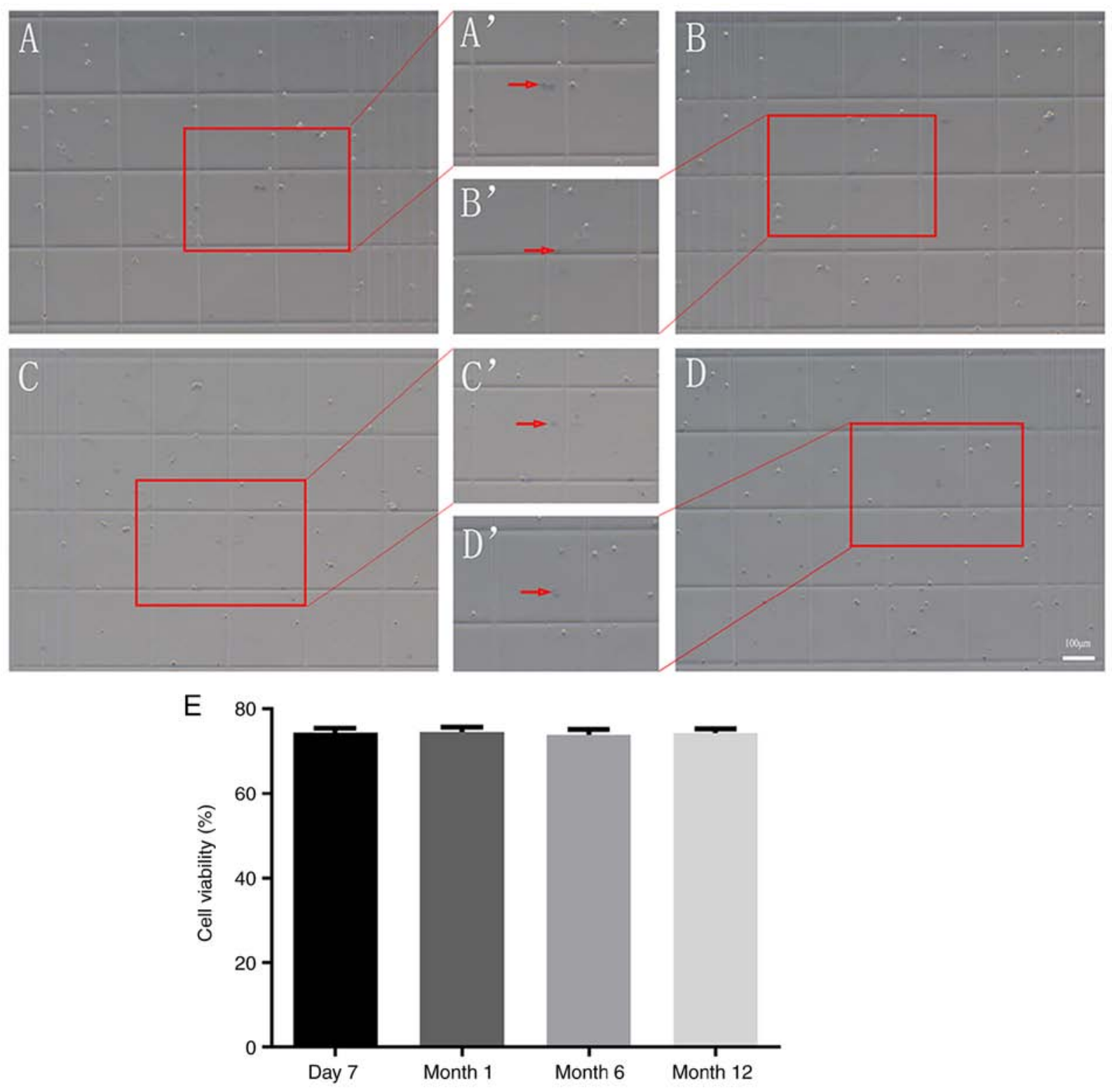

Figure 5. Neural stem cell viability. Cell viability following cryopreservation for (A) 7 days, (B) 1 month, (C) 6 months and (D) 12 months was measured. (A'-D') High magnification images of the area labeled with red boxes in parts (A-D) (magnification, $x 2$ ). Arrow indicates necrotic cells. (E) NSCs cryopreserved for 7 days, or 1, 6 or 12 months did not show any differences in viability (all $\mathrm{P}>0.05$ ). Scale bar=100 $\mu \mathrm{m}$.

cells between non-cryopreserved and cryopreserved NSCs (all $\mathrm{P}>0.05$ ) or among NSCs cryopreserved for 7 days, or 1,6 or 12 months (all $\mathrm{P}>0.05$ ).

Multipotent differentiation after cryopreservation. The differentiation potential of NSCs following different durations of cryopreservation was analyzed. As demonstrated in Fig. 7, all non-cryopreserved and cryopreserved NSCs were able to differentiate into astrocytes, neurons and oligodendrocytes. In addition, there were no significant differences between non-cryopreserved and cryopreserved NSCs (all P>0.05) or among NSCs cryopreserved for 7 days, or 1,6 or 12 months (all $\mathrm{P}>0.05$ ).

Determination of the cell diameter following cryopreservation. To assess the self-renewal capability of NSCs following different cryopreservation durations, non-cryopreserved and cryopreserved secondary NSCs were dissociated and cultured in SFM. As indicated in Fig. 8, cryopreserved NSCs formed neurospheres identical to those of non-cryopreserved NSCs (all $\mathrm{P}>0.05$ ). In addition, NSCs cryopreserved for 7 days, or 1,6 or 12 months did not show significant differences in cell diameter (all $\mathrm{P}>0.05$ ).

\section{Discussion}

The present study detailed a modified technique for the isolation, culture and cryopreservation of rat embryonic NSCs. The viability, nestin expression, multipotentiality and diameter of non-cryopreserved NSCs and NSCs cryopreserved for 7 days, or 1,6 or 12 months were evaluated. Rat embryonic NSCs were successfully obtained, and they maintained the capability for self-renewal and multipotent differentiation even after long-term cryopreservation (up to 12 months). 


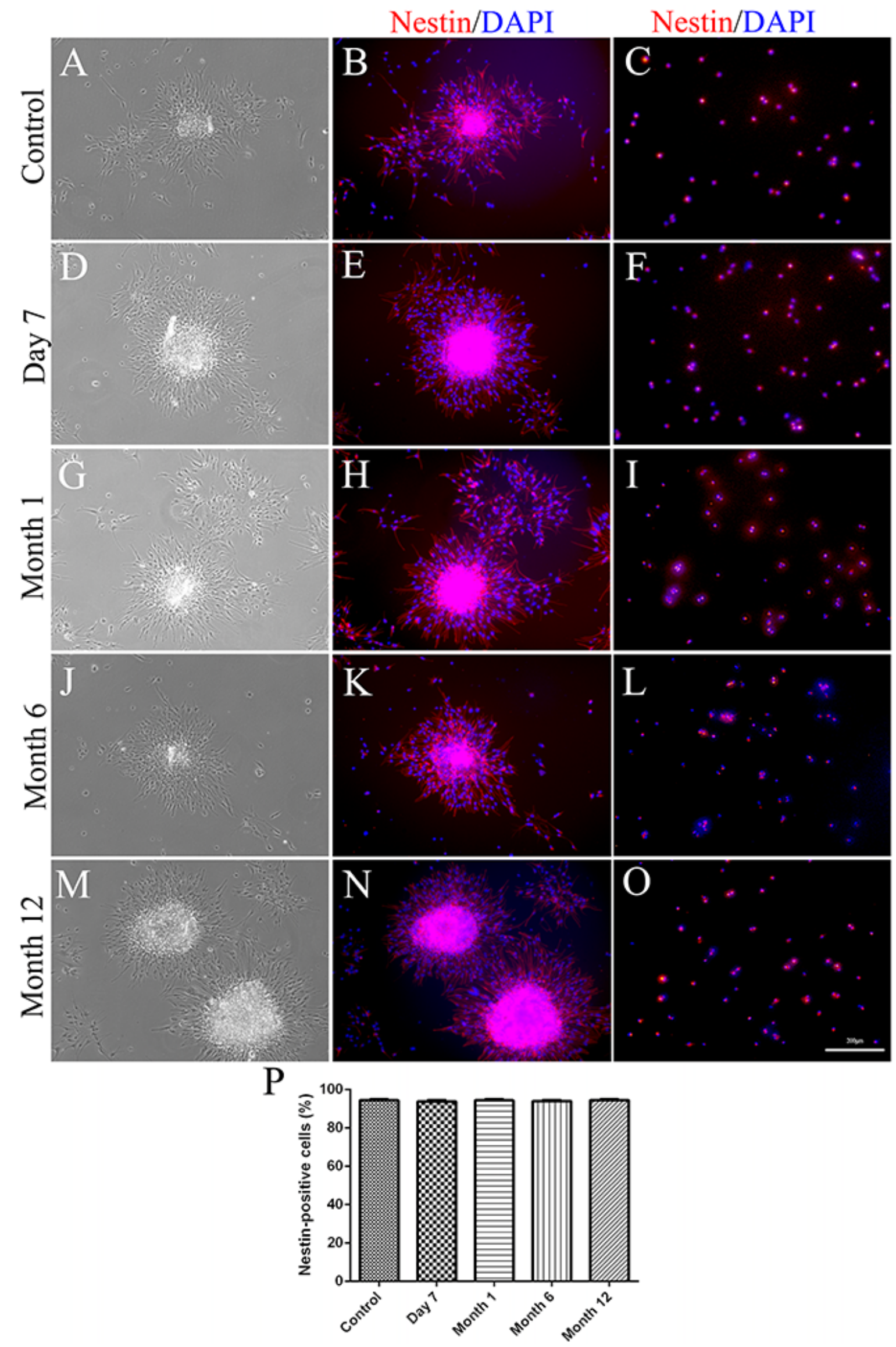

Figure 6. (A-P) Nestin expression in neurospheres. (A, D, G, J and M) Phase contrast micrograph of non-cryopreserved and cryopreserved secondary neurospheres. (B, E, H, K and N) Expression of nestin in non-cryopreserved and cryopreserved secondary neurospheres. (C, F, I, L and O) Expression of nestin in dissociated neurospheres. (P) Quantification of immunostaining data. No significant differences were observed in the percentage of nestin-positive cells between non-cryopreserved and cryopreserved secondary neurospheres (all P>0.05) or among NSCs cryopreserved for 7 days, or 1,6 or 12 months (all $\mathrm{P}>0.05$ ). Scale bar $=100 \mu \mathrm{m}$. 


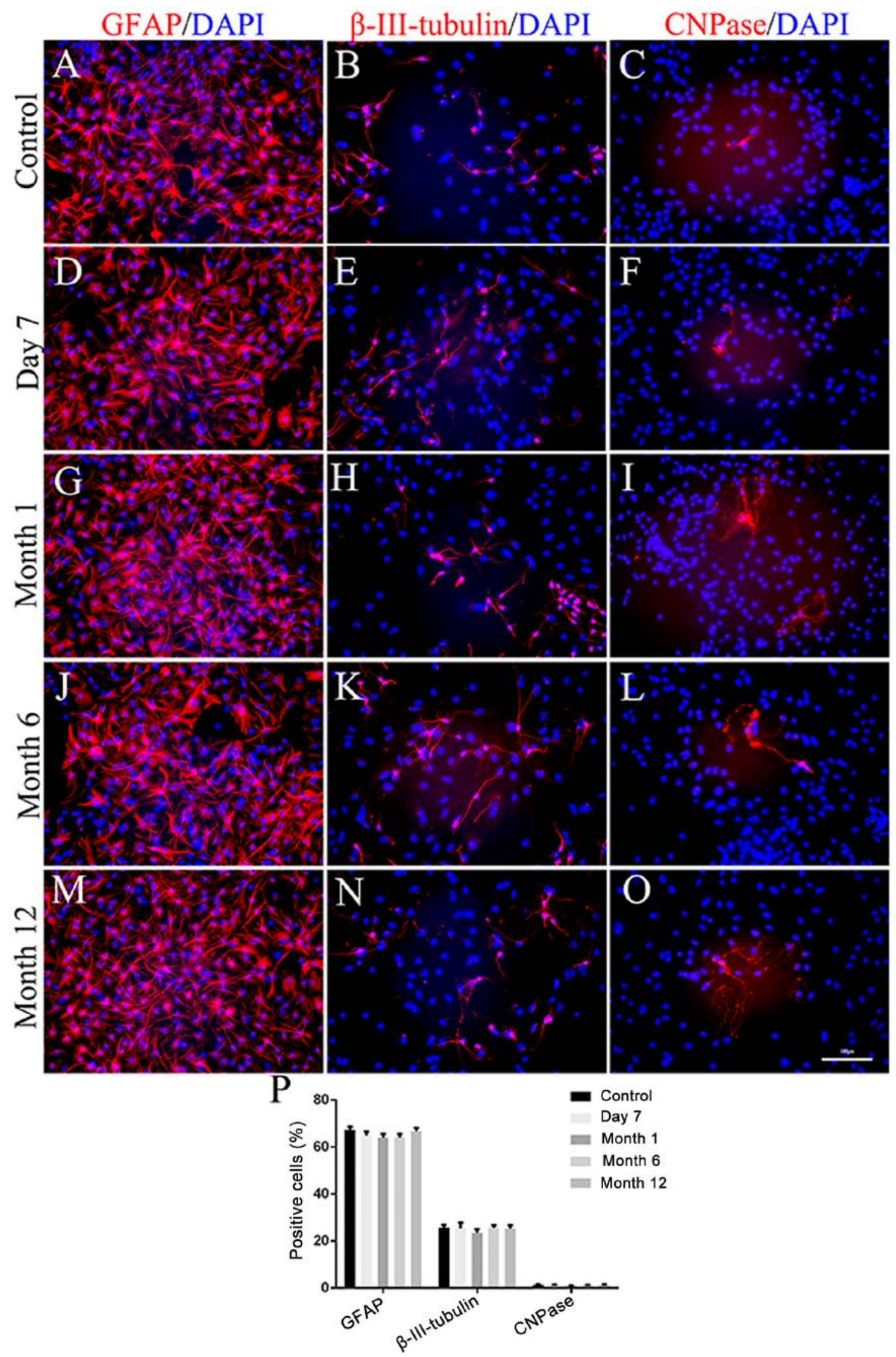

Figure 7. (A-P) Differentiation capacity of neurospheres. (A, D, G, J and M) Representative image immunocytochemical staining for GFAP (red) and DAPI (blue) in non-cryopreserved and cryopreserved neurospheres-derived astrocytes. (B, E, H, K and N) Representative image of immunocytochemical staining for $\beta$-III-tubulin (red) and DAPI (blue) in non-cryopreserved and cryopreserved neurospheres-derived neurons. (C, F, I, L and O) Representative image of immunocytochemical staining for CNPase (red) and DAPI (blue) in non-cryopreserved and cryopreserved neurospheres-derived oligodendrocytes. (P) Quantification of immunostaining data. No significant differences were observed between non-cryopreserved and cryopreserved secondary neurospheres (all $\mathrm{P}>0.05$ ) or among NSCs cryopreserved for 7 days, or 1,6 or 12 months (all P>0.05). Scale bar $=100 \mu \mathrm{m}$. GFAP, glial fibrillary acidic protein; $\beta$-III-tubulin, neuron-specific class III beta-tubulin; CNPase, cyclic nucleotide phosphodiesterase. 
A

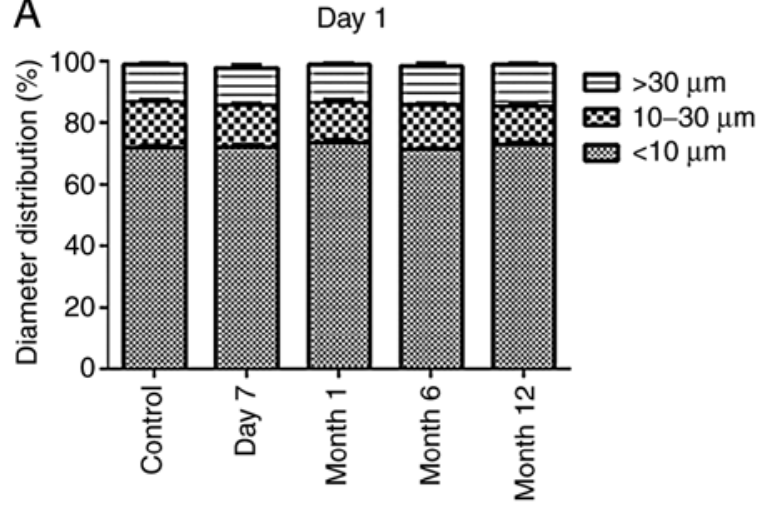

C

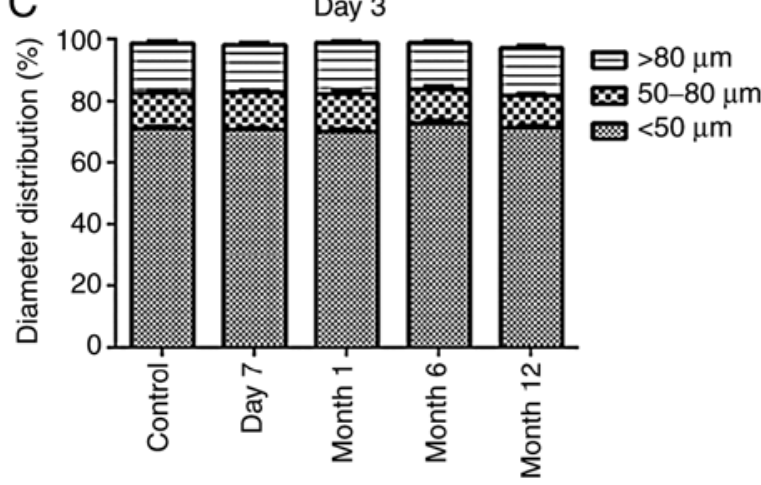

B

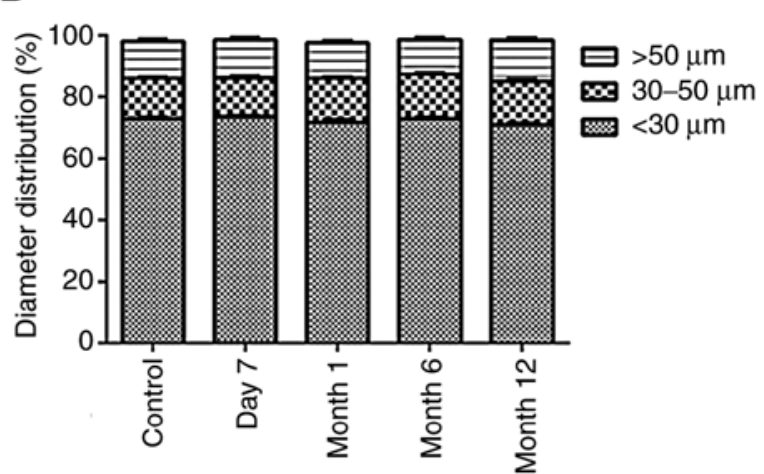

Day 4

D

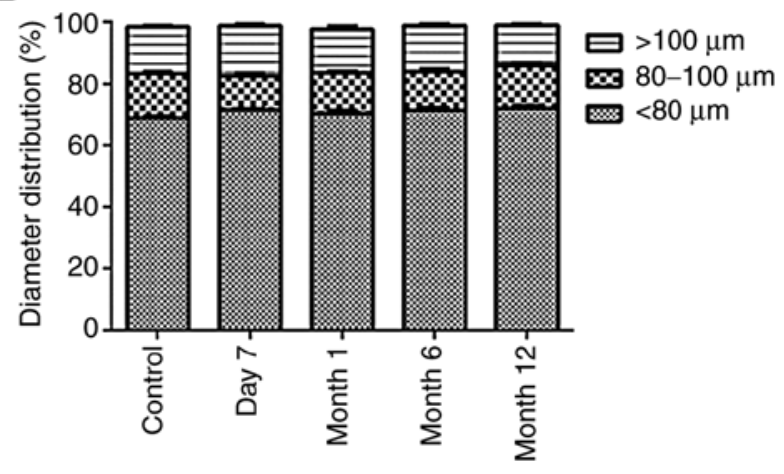

Figure 8. Determination of the cell diameter following cryopreservation. (A-D) Diameters of non-cryopreserved and cryopreserved secondary neurospheres after (A) 1, (B) 2, (C) 3 and (D) 4 days of culture in serum-free medium. No significant differences in cell diameter between neural stem cells cryopreserved for 7 days, or 1,6 or 12 months were observed (all $\mathrm{P}>0.05$ ).

Compared with other protocols, 2 important skills were modified in the protocol of the present study: Colorless high-glucose medium and a sequential digestion strategy. Firstly, NSCs are cells with a high metabolism, and they are easily damaged upon exposure to a sugar-free environment, which may not provide sufficient energy and therefore result in decreased cell viability. In previous studies, PBS $(21,25)$, Hank's balanced salt solution (HBSS) $(7,17)$ or Leibovitz's L-15 $(10,15)$ were used, but in the present study, DMEM-HG was chosen as a temporary storage solution to provide energy. Furthermore, DMEM-HG is colorless (without phenol red) and may provide a clear operative view for tissue dissection (17), which is particularly important during the process of pia mater and blood vessel removal. In addition, $1 \% \mathrm{P} / \mathrm{S}$ was added to the DMEM-HG to decrease potential contamination during the relatively long dissection procedure $(10,26)$. Secondly, a strategy of sequential digestion was applied in the present study. Following the initial digestion (a combination of enzymatic dissociation by accutase/DNase I and mechanical pipetting during enzymatic dissociation), undissociated tissues settled at the bottom of the tube while single cells were suspended in the upper phase after standing for $2 \mathrm{~min}$. Suspended cells were collected for temporary storage to prevent over-digestion, and the remaining undissociated tissues were collected for a secondary digestion to ensure the maximal harvest of NSCs $(11,12,19)$. The sequential digestion method minimizes the probability of excessive dissociation and thereby promotes NSCs survival and viability compared with the previous one-step digestion protocols used by a number of previous studies $(23,27)$. In addition, a $35 \mathrm{~mm}$ Petri dish was applied for tissue digestion rather than a centrifuge tube or a microtube, which was used by numerous previous studies $(23,27,28)$. This approach allowed tissue pieces to be well-distributed in the enzymatic solution, which not only facilitated close contact between the tissues and enzymes but also allowed for improved monitoring of the digestion status under a microscope (12).

To obtain high-viability NSCs, tissue collection and cell isolation should be performed as quickly as possible (within $2 \mathrm{~h}$ ), not only because tissues become viscous and pulpy, leading to increased difficulty of dissection, but also due to the fact that the viability of NSCs decreases over time $(2,11,25,29,30)$. In addition, low-temperature strategies were applied throughout the dissection process to enhance cell viability $(8,10,12,21,23,31)$. It was identified to decrease the tissue consumption of oxygen and energy, which is crucial for the survival of the isolated NSCs $(7,10,12,23)$. Therefore, the temperature was maintained at a low level during tissue dissection; for example, $70 \%$ ethanol at $-20^{\circ} \mathrm{C}$ was used for disinfection and to rapidly decrease the embryo temperature. Dishes containing $4^{\circ} \mathrm{C}$ DMEM-HG with $1 \% \mathrm{P} / \mathrm{S}$ were placed on ice for tissue dissection, and centrifuge tubes and dishes containing $4^{\circ} \mathrm{C}$ DMEM-HG with $1 \% \mathrm{P} / \mathrm{S}$ were placed on ice or under refrigeration for tissue preservation. In addition to the aforementioned noes for the dissection procedure, complete and thorough removal of the pia mater and blood vessels is important $(10,12,16)$. The presence of the pia mater and blood vessels may increase the force required for pipetting, ultimately 
increasing the mechanical damage to the NSCs $(10,12,16)$. In addition, pia mater and blood vessels are the primary sources of contaminating cells, for example endothelial cells, fibroblasts and blood cells $(10,12,16)$. If they are not completely removed, these cells may overgrow and decrease the purity of the NSC culture.

The tissue digestion procedure is one of the most critical steps for obtaining a high yield of NSCs. If not handled properly, this process may result in excessive cell death $(7,10,16,30)$. Notably, smaller tissue pieces increase the cell dissociation efficiency, primarily due to the increased contact area between the tissue and dissociation enzyme solutions. In addition, the potential for mechanical damage by increased pipetting force is decreased $(7,10,16,30)$. Therefore, dissected tissues were minced into small pieces with microscissors prior to commencing the digestion procedure. However, the mincing step should not be performed for extended periods of time; the optimal duration is $\sim 1 \mathrm{~min}$. The efficiency of mincing may be improved with practice and the aid of a stereomicroscope. Tissue digestion involves enzymatic and mechanical methods $(30,31)$. The enzymatic treatment is the application of proteolytic enzymes, including trypsin-EDTA and accutase, which degrade the highly structured extracellular matrix surrounding NSCs to isolate them from the rest of the tissue (30). Trypsin-EDTA has been employed in several studies $(17,21-23,26,31)$, However, it is difficult to control the enzymatic reaction time, which may affect sphere formation, cause cells to attach to the substrate and differentiate, or result in massive cell death. Accutase is a less aggressive enzyme $(17,19,21-23)$ that has been demonstrated to increase cell survival compared with that associated with trypsin-EDTA and does not require FBS. By contrast, trypsin-EDTA is usually inactivated by adding FBS; the latter may affect NSC differentiation and introduce contaminants $(26,30)$. In addition to accutase, DNase I is always added to break down the accumulated mass of sticky DNA released from damaged cells, which prevents subsequent dissociation $(18,30)$.

During or following enzymatic dissociation, mechanical dissociation is applied. This relatively aggressive method is accomplished using either a fire-polished bevel tip glass pipette, a commercially available disposable plastic pipette or a sterile syringe needle that breaks up tissue pieces by pushing them against the bottom/base or side of the tube/plate $(19,30)$. Of note, during mechanical digestion, the formation of air bubbles should be avoided as an excess of bubbles decreases the cell viability and trituration efficiency and increases the risk of contamination $(2,7,10,16-19,21,23)$. Compared with enzymatic digestion, mechanical dissociation alone takes more time, requires an increased level of experimental skills, induces more variability (digestion efficiency depends on an experimenter's experience), and causes more cell aggregation and cell death $(11,19,21,22,30)$. Therefore, a combination of enzymatic dissociation by accutase/DNase I and mechanical pipetting during enzymatic dissociation is considered o be one of the best procedures to achieve sufficient tissue dissociation with minimal cell death.

Following tissue digestion and cell plating, the growth of NSCs was closely monitored. A total of one-half of the medium was replaced with new medium after 3 days of culture. The mixed medium contained pro-survival factors produced by cells and released into the conditioning medium (10). A complete medium change was performed during cell passaging after 7-8 days of culture, when the majority of neurospheres were $<150-200 \mu \mathrm{m}$ in diameter. Larger neurospheres are difficult to dissociate and eventually begin to differentiate $(2,11,16,30,32)$. In addition, certain cells in the core of excessively large neurospheres are susceptible to death due to a lack of access to oxygen and nutrients, as they are not in direct contact with the culture medium $(2,11,16,30,32)$.

To characterize the multipotent ability of the NSCs, the cells were exposed to 1 type of medium (DMEM/F-12 medium containing B-27 supplement and 1\% FBS), not 3 different media, for example: Retinoic acid plus forskolin for neurons induction; insulin-like growth factor-1 for oligodendrocytes induction; and leukemia inhibitory factor with bone morphogenic protein-2 for astrocytes induction, as described previously $(33,34)$. The difference between the methods involving 1 medium and 3 media lies in the final different differentiation rate of neurons, oligodendrocytes and astrocytes; the 1 medium method has been applied by a number of previous studies to illustrate the different function of drugs or materials on NSCs $(35,36)$. In addition, if 3 different media are used, more stimulation factors, for example retinoic acid and forskolin, are added in the media; and different time periods are required for neuron (4 days), astrocyte (4 days), and oligodendrocyte (6 days) differentiation $(33,34)$. Consequently, incubation time becomes an influencing factor; the results of the present study may have been affected by these additional factors. For the present study, the multipotent character and recovery ability of the cryopreserved NSCs, not the different effect of stimulation factors and time period, were the focus. Therefore, the 1 medium method was chosen to examine our hypothesis. Future studies should also apply the 3 media protocol to characterize the multipotent ability of NSCs in more detail.

Using the procedure described, the present study successfully obtained highly viable rat embryonic NSCs that proliferated, expressed nestin and differentiated into neurons, astrocytes and oligodendrocytes. However, these cells have a limited expansion period in vitro and frequently exhibit spontaneous differentiation. Therefore, NSCs are commonly cryopreserved for future research and transplantation $(24,37)$. The NSC state (single-cell suspension or NSC spheres), freezing medium composition and freezing cell density are the major determinants of NSC survival and viability following the thawing process $(15,16)$. In the present study, small spheres in the logarithmic phase $24 \mathrm{~h}$ after the secondary passage were collected for cryopreservation. A high sphere density $\left(5-10 \times 10^{6}\right.$ cells $\left./ \mathrm{ml}\right)$ was used during freezing to enhance sphere re-establishment following cryopreservation, based on data from previous studies $(7,16,30)$. SFM supplemented with $10 \%$ DMSO was used as the freezing medium, and FBS was not added to the freezing medium considering its induction effect on NSC differentiation and the chance of introducing contaminants $(4,15,16,30)$. Furthermore, it is critical to remove all the freezing solution from the frozen pellet during recovery and re-rinse the cells using DMEM-HG, as the introduction of DMSO into the culture medium is cytotoxic $(7,16,30)$. Therefore, the thawed NSCs were rinsed twice using $40 \mathrm{ml}$ DMEM-HG, and the entire supernatant was discarded 
following centrifugation. In addition, the effect of different cryopreservation durations on rat embryonic NSCs was explored. Cells were thawed after 7 days, or 1, 6 or 12 months of storage in liquid nitrogen, and the viability, stemness state, self-renewal ability and multipotency of differentiation were determined. The results suggested that a number of the cells retained their viability following frozen storage and that the viability of NSCs cryopreserved for different time periods was similar. NSCs cryopreserved for different periods of time maintained stemness and the ability to self-renew and differentiate into astrocytes, neurons and oligodendrocytes.

The present study contained a few limitations that should be addressed. Firstly, more immunostaining markers of NSCs, including transcription factor SOX-2 (SOX2), RNA-binding protein Musashi homolog 1 (MUSASHI-1) and prominin-1 $(\mathrm{CD} 133+)$, were not used to verify their nature. Subsequent studies should perform SOX2, MUSASHI-1 and CD133+ immunostaining. Secondly, only 1 medium was used to characterize the multipotent ability of NSCs; the use of 3 media concomitantly would address this point in greater detail in future studies.

In conclusion, the in vitro culture of rat embryonic NSCs has been widely applied for the investigation of the cellular and molecular mechanisms underlying neurogenesis and brain regeneration. The present study described a modified primary culture protocol for successfully obtaining rat embryonic NSCs that maintain their ability to self-renew and multipotency after different time periods of cryopreservation.

\section{Acknowledgements}

Not applicable.

\section{Funding}

The present study was supported by grants from the National Natural Science Foundation of China (grant no., 81501899), the State Key Program of the National Natural Science Foundation of China (grant nos., 81330042 and 81620108018), the Special Program for Sino-Russian Joint Research Sponsored by the Ministry of Science and Technology, China (grant no., 2014DFR31210), the Key Program Sponsored by the Tianjin Science and Technology Committee, China (grant nos., 13RCGFSY19000 and 14ZCZDSY00044), the Science Foundation of Tianjin Medical University for Young Scholars (grant no., 2014KYQ01), and the Science Foundation of Tianjin Medical University General Hospital for Young Scholars (grant no., ZYYFY2014037).

\section{Availability of data and materials}

All data generated or analyzed during the present study are included in this published article.

\section{Authors' contributions}

HZ, HY, LLu and XL designed and performed the experiments, analyzed the data and co-wrote the article. BP, ZF, TC, JL, YK and LLiu analyzed the data and edited the manuscript. GN, WYD and PW designed the study and critically revised the article for important intellectual content. XK and SF designed the experiments, provided materials and funding and co-wrote the article. All authors agree that all the questions related to the accuracy or integrity of the paper have been appropriately investigated and resolved and have given final approval of the version to be published.

\section{Ethics approval and consent to participate}

The present study was performed with the approval of the Ethics Committee of Tianjin Medical University and complied with the U.S. National Institutes of Health Guide for the Care and Use of Experimental Animals and the Society for Neuroscience Use of Animals in Neuroscience Research Guidelines.

\section{Patient consent for publication}

Not applicable.

\section{Competing interests}

The authors declare that they have no competing interests.

\section{References}

1. Schiefer HB: Guide to the care and use of experimental animals, volume 2. Can Vet J 26: 59, 1985.

2. OlferdB E, Cross B, McWillian D and Mc Willian A: Guidelines for the use of animal in neuroscience research. Canadian Council on Care (CCAC), Ottawa, Canada 163-165, 1997.

3. Reynolds BA, Tetzlaff $\mathrm{W}$ and Weiss S: A multipotent EGFresponsive striatal embryonic progenitor cell produces neurons and astrocytes. J Neurosci 12: 4565-4574, 1992.

4. Rietze RL and Reynolds BA: Neural stem cell isolation and characterization. Methods Enzymol 419: 3-23, 2006.

5. Chojnacki A and Weiss S: Production of neurons, astrocytes and oligodendrocytes from mammalian CNS stem cells. Nat Protoc 3: 935-940, 2008.

6. Gritti A, Galli R and Vescovi AL: Clonal analyses and cryopreservation of neural stem cell cultures. Methods Mol Biol 438: 173-184, 2008

7. Revishchin AV, Korochkin LI, Okhotin VE and Pavlova GV: Neural stem cells in the mammalian brain. Int Rev Cytol 265: 55-109, 2008.

8. Ahmed S: The culture of neural stem cells. J Cell Biochem 106: $1-6,2009$.

9. Guo W, Patzlaff NE, Jobe EM and Zhao X: Isolation of multipotent neural stem or progenitor cells from both the dentate gyrus and subventricular zone of a single adult mouse. Nat Protoc 7: 2005-2012, 2012.

10. Kumar K, Singh R, Kumar M, Agarwal P, Mahapatra PS, Kumar A, Malakar D and Bag S: Isolation and characterization of neural stem cells from buffalo. Int J Neurosci 124: 450-456, 2014.

11. Huang H, Mao G, Chen L and Liu A: Progress and challenges with clinical cell therapy in neurorestoratology. J Neurorestoratol 3: 91-95, 2015.

12. Hameed LA and Simon A: Isolation and culture of neurospheres from the adult newt brain. Methods Mol Biol 1290: 197-204, 2015.

13. Louis SA and Reynolds BA: Generation and differentiation of neurospheres from murine embryonic day 14 central nervous system tissue. Methods Mol Biol 290: 265-280, 2005.

14. Xu SY, Wu YM, Ji Z, Gao XY and Pan SY: A modified technique for culturing primary fetal rat cortical neurons. J Biomed Biotechnol 2012: 803930, 2012.

15. Ahlenius H and Kokaia Z: Isolation and generation of neurosphere cultures from embryonic and adult mouse brain. Methods Mol Biol 633: 241-252, 2010. 
16. Pacey L, Stead S, Gleave J, Tomczyk K and Doering L: Neural stem cell culture: Neurosphere generation, microscopical analysis and cryopreservation. Nat Protoc 1: 215-222, 2006.

17. Bonner JF, Haas CJ and Fischer I: Preparation of neural stem cells and progenitors: Neuronal production and grafting applications. Methods Mol Biol 1078: 65-88, 2013.

18. Brewer GJ and Torricelli JR: Isolation and culture of adult neurons and neurospheres. Nat Protoc 2: 1490-1498, 2007.

19. Louis SA, Mak CK and Reynolds BA: Methods to culture, differentiate, and characterize neural stem cells from the adult and embryonic mouse central nervous system. Methods Mol Biol 946: 479-506, 2013.

20. Ortega F, Berninger B and Costa MR: Primary culture and live imaging of adult neural stem cells and their progeny. Methods Mol Biol 1052: 1-11, 2013.

21. Bizy A and Ferrón SR: Isolation, long-term expansion, and differentiation of murine neural stem cells. Methods Mol Biol 1212: 103-112, 2015

22. Lopez-Ramirez MA, Calvo CF, Ristori E, Thomas JL and Nicoli S: Isolation and culture of adult zebrafish brain-derived neurospheres. J Vis Exp 53617, 2016.

23. Aligholi H, Hassanzadeh G, Gorji A and Azari H: A novel biopsy method for isolating neural stem cells from the subventricular zone of the adult rat brain for autologous transplantation in CNS injuries. Methods Mol Biol 1462: 711-731, 2016.

24. Hinsch K and Zupanc GK: Isolation, cultivation, and differentiation of neural stem cells from adult fish brain. J Neurosci Methods 158: 75-88, 2006.

25. Belenguer G, Domingo-Muelas A, Ferrón SR, Morante-Redolat JM and Fariñas I: Isolation, culture and analysis of adult subependymal neural stem cells. Differentiation 91: 28-41, 2016.

26. Hitoshi S, Kippin T and van der Kooy D: Culturing adult neural stem cells: Application to the study of neurodegenerative and neuropsychiatric pathology. In: Neurogenesis in the adult brain II: Clinical implications. Seki T, Sawamoto K, Parent JM and Alvarez-Buylla A (eds.) Springer, Tokyo, pp189-207, 2011.
27. Annese T, Corsi P, Ruggieri S, Tamma R, Marinaccio C,Picocci S, Errede M, Specchia G, De Luca A, Frassanito MA, et al: Isolation and characterization of neural stem cells from dystrophic $\mathrm{mdx}$ mouse. Exp Cell Res 343: 190-207, 2016.

28. Hugnot JP: Isolate and culture neural stem cells from the mouse adult spinal cord. Methods Mol Biol 1059: 53-63, 2013.

29. Ferrari D, Binda E, De Filippis L and Vescovi AL: Isolation of neural stem cells from neural tissues using the neurosphere technique. Curr Protoc Stem Cell Biol 2: 2D.6, 2010.

30. Oliver-De la Cruz J and Ayuso-Sacido A: Neural stem cells from mammalian brain: Isolation protocols and maintenance conditions. In: Neural Stem Cells and Therapy. Sun T (ed.) Intech, Rijeka, pp3-30, 2012.

31. Azari H, Rahman M, Sharififar S and Reynolds BA: Isolation and expansion of the adult mouse neural stem cells using the neurosphere assay. J Vis Exp 2393, 2010.

32. Siebzehnrubl FA, Vedam-Mai V, Azari H, Reynolds BA and Deleyrolle LP: Isolation and characterization of adult neural stem cells. Methods Mol Biol 750: 61-77, 2011.

33. Hsieh J, Nakashima K, Kuwabara T, Mejia E and Gage FH: Histone deacetylase inhibition-mediated neuronal differentiation of multipotent adult neural progenitor cells. Proc Natl Acad Sci USA 101: 16659-16664, 2004.

34. Hsieh J, Aimone JB, Kaspar BK, Kuwabara T, Nakashima K and Gage FH: IGF-I instructs multipotent adult neural progenitor cells to become oligodendrocytes. J Cell Biol 164: 111-122, 2004.

35. Li JY, Liu J, Manaph NPA, Bobrovskaya L and Zhou XF: ProBDNF inhibits proliferation, migration and differentiation of mouse neural stem cells. Brain Res 1668: 46-55, 2017.

36. Shirazi HA, Rasouli J, Ciric B, Rostami A and Zhang GX: 1,25-Dihydroxyvitamin D3 enhances neural stem cell proliferation and oligodendrocyte differentiation. Exp Mol Pathol 98: 240-245, 2015.

37. Ma XH, Shi Y, Hou Y, Liu Y, Zhang L, Fan WX, Ge D, Liu TQ and Cui ZF: Slow-freezing cryopreservation of neural stem cell spheres with different diameters. Cryobiology 60: 184-191, 2010. 